\title{
Early changes in longitudinal performance predict future improvement in global left ventricular function during long term $\beta$ adrenergic blockade
}

\author{
B Andersson, F Waagstein, K Caidahl, I Eurenius, M Scharin Täng, R Wikh
}

\begin{abstract}
Objective-Contraction of longitudinal and subendocardial myocardial muscle fibres is reflected in descent of the atrioventricular (AV) plane. The aim was therefore to determine whether $\beta$ blocker treatment with prolongation of diastole might result in improved function as reflected by AV plane movements in patients with chronic heart failure.

Design-Double blind, randomised, placebo controlled and open intervention study.

Setting-University hospital.

Patients-Patients with congestive heart failure: placebo controlled $(\mathrm{n}=26)$ and an open proto$\operatorname{col}(\mathrm{n}=15)$.

Interventions -12 months of metoprolol treatment.

Main outcome measures-Short axis and long axis echocardiography, invasive haemodynamics, radionuclide angiography.

Results-Recovery of systolic and diastolic function during metoprolol treatment was reflected by early changes in mean (SD) AV plane amplitude, from 5.3 (2.0)\% to 7.1 (3.2)\% and $7.8(3.1) \%$ (at 3 and 12 months, respectively; $\mathrm{p}<0.05$ ). In a multivariate analysis, only the change in AV plane amplitude by three months was independently associated with improvement in pulmonary capillary wedge pressure by six months $(r=0.80, \mathrm{p}=0.017)$. Change in AV plane amplitude by three months was also a better predictor of improvement in ejection fraction by 12 months $(r=0.78, \mathrm{p}<0.001)$ than changes in radionuclide ejection fraction by three months $(\mathrm{r}=0.34, \mathrm{p}=0.049)$.

Conclusions-Improvement in longitudinal contraction was closely associated with a decrease in left ventricular filling pressure during metoprolol treatment. This association was stronger than changes in short axis performance or radionuclide ejection fraction, emphasising the importance of AV plane motion for left ventricular filling and systolic performance in patients with heart failure.

(Heart 2000;84:599-605)
\end{abstract}

Keywords: diastolic function; metoprolol; dilated cardiomyopathy; echocardiography

Following the convincing results of the two major survival studies, the cardiac insufficiency bisoprolol study II (CIBIS-II) and the metoprolol CR/XL randomised intervention trial in congestive heart failure (MERIT-HF), ${ }^{12}$ adrenergic $\beta$ blockers have now become part of the accepted therapeutic arsenal in the treatment of patients with congestive heart failure. Consistent findings of improvement in systolic function have been reported in numerous trials. Through the reduction in heart rate, $\beta$ blockers also have prominent diastolic effects. However, studies on diastolic function and $\beta$ blocker treatment are sparse, and clear cut positive results are still lacking. ${ }^{3}$

Studies of left ventricular function have primarily been directed at circumferential movement, and less attention has been paid to the longitudinal contraction pattern. It has been proposed that the movements of the fibrous structure in the atrioventricular (AV) plane are dependent on longitudinal subendocardial myocardial fibres in particular, and would therefore reflect regional ischaemia and diastolic function. ${ }^{45}$ Recordings of these movements have provided data closely related to long term mortality in congestive heart failure ${ }^{6}$ Furthermore, the AVP motion reflects left atrial volume changes, which may provide information about the function of this chamber as well. ${ }^{7}$ Most studies of the longitudinal movements in the AV plane have used $M$ mode echocardiography, but investigations with magnetic resonance imaging and tissue Doppler have also shown similar results in terms of absolute amplitudes in normal individuals. ${ }^{8}$

This study was a predefined substudy of the metoprolol in dilated cardiomyopathy (MDC) trial, intended to study the effects of long term metoprolol treatment on long axis and short axis motion in patients with congestive heart failure, and focusing on the longitudinal contraction pattern. We hypothesised that the contraction pattern in the AV plane is important for cardiac pump function in patients with heart failure, and it should be possible to observe recovery during $\beta$ blocker treatment by recording movements in this plane.

\section{Methods}

The patients in the present study were in part recruited from the MDC trial, and the study was prospectively planned as a substudy of the main trial. The main study enrolled 383 patients in a double blind, randomised, placebo controlled trial, stratified according to investi- 
gating centre and ejection fraction $(<20 \%$ or $20-39 \%$, respectively). ${ }^{10}$ Patients with symptomatic idiopathic dilated cardiomyopathy and an ejection fraction of $<40 \%$ were eligible for this study. Exclusion criteria were: coronary artery disease confirmed by coronary arteriography, systemic disease, insulin dependent diabetes mellitus, excessive alcohol consumption, hypertension, signs of active myocarditis, and other serious disease that might influence the prognosis of the patient. In the present substudy, performed at the coordinating centre at Sahlgrenska University Hospital, 14 patients were randomised to metoprolol, and 12 patients were randomised to placebo.

To increase the number of patients treated with $\beta$ blockade, 15 patients were enrolled in an open protocol, parallel to the placebo controlled MDC trial, with titration schedule and follow up similar to the controlled trial. All these 15 patients presented with an echocardiographic picture of dilated cardiomyopathy, with global left ventricular dysfunction and without regional hypokinesis, but during clinical investigation it was discovered that the patients met an exclusion criterion in the MDC trial. The reasons for not including these patients in the double blind study were: ejection fraction above $40 \%$ in two, ischaemic cardiomyopathy in six, insulin dependent diabetes mellitus in one, systemic collagenosis in one, muscular dystrophy in one, and a recently completed placebo period in a previous drug trial in four. Baseline characteristics are given in table 1 . Treatment with metoprolol was continued with slowly increasing doses from $5 \mathrm{mg}$ twice daily to $50 \mathrm{mg}$ three times daily, as previously described. ${ }^{11}$ The period of treatment was 12 months, and investigations were performed at baseline and after three, six, and 12 months of treatment.

INVASIVE HAEMODYNAMIC AND RADIONUCLIDE INVESTIGATIONS

Right heart catheterisation was performed in nine metoprolol and nine placebo patients in the double blind protocol. The procedure was performed at baseline and after six and 12 months of follow up. ${ }^{11}$ Equilibrium radionuclide angiocardiography was performed at rest, and at three, six, and 12 months of follow up, as described elsewhere. ${ }^{12}$
ECHOCARDIOGRAPHY

Cross sectional echocardiography was performed with the patient in the left lateral position, and recordings were obtained during relaxed end expiratory apnoea. Transverse short axis $M$ mode recordings of the left ventricle were obtained from the parasternal view, according to the recommendations of the American Association of Echocardiography. ${ }^{13}$ Longitudinal contractions were recorded by directing the $M$ mode beam from the apex to the fibrous tissue of the AV plane at the angle of the proximal left ventricular wall and the insertion of the mitral valve apparatus. The ultrasound beam was adjusted to obtain a continuous echo without interfering echoes from the mitral valve. The recordings were performed in the standard apical four chamber view, and those of the septal and the left lateral portions of the AV plane were obtained with magnification of the structures (using zoom function). The AV plane movements were recorded on videotape and on strip charts at $100 \mathrm{~mm} / \mathrm{s}$. Recordings of the pericardial contour of left ventricular apex in relation to the thoracic wall were made using zoom. Mitral flow was determined by pulsed wave Doppler with the sampling volume between the tips of the mitral valve in the four chamber view. An ECG signal was reproduced on all echocardiographic tracings.

\section{MEASUREMENTS}

Evaluation and calculations of $M$ mode tracings were performed by two independent investigators who were blinded to the patients' clinical data. Short axis $M$ mode recordings were analysed on a digitising board with tracing of posterior wall and interventricular septal contours. The AV plane movements were digitised, as was the epicardial contour of the apex. Data acquisition was achieved by a software program (CAS cardiac analysis software), developed in-house. The program generated calculations of left ventricular dimensions, wall motion velocities, maximum speed of contraction and relaxation, and time intervals of the short axis and long axis movements.

Six points in the curve of the AV plane were identified: (1) the start of systolic contraction; (2) the end of systolic contraction; (3) the start of rapid filling; (4) the end of rapid filling; (5) the start of atrial contraction; and (6) the end of atrial contraction (fig 1). The systolic AV

Table 1 Baseline characteristics in 41 patients with congestive heart failure

\begin{tabular}{|c|c|c|c|c|c|c|c|}
\hline & \multicolumn{3}{|c|}{ Double blind study } & \multicolumn{2}{|l|}{ Open study } & \multirow[b]{2}{*}{$\begin{array}{l}\text { All metoprolol } \\
(n=29)\end{array}$} & \multirow[b]{2}{*}{$p$ Valu } \\
\hline & $\begin{array}{l}\text { Placebo } \\
(n=12)\end{array}$ & $\begin{array}{l}\text { Metoprolol } \\
(n=14)\end{array}$ & $p$ Value & $\begin{array}{l}\text { Metoprolol } \\
(n=15)\end{array}$ & $p$ Value & & \\
\hline Age (years) & $56(6.3)$ & $46(14)$ & $<0.05$ & $55(10)$ & NS & $51(13)$ & NS \\
\hline Sex $($ male, $\%)$ & 67 & 71 & NS & 80 & NS & 76 & NS \\
\hline \multicolumn{8}{|l|}{ NYHA functional class (n) } \\
\hline II/III/IV & $1 / 10 / 1$ & $3 / 11 / 0$ & NS & $0 / 15 / 0$ & NS & $3 / 26 / 0$ & NS \\
\hline Ejection fraction (\%) & $25(7.2)$ & $25(7.7)$ & NS & $30(12)$ & NS & $28(11)$ & NS \\
\hline Heart rate (beats/min) & $88(14)$ & $85(17)$ & NS & $79(20)$ & NS & $82(18)$ & NS \\
\hline ACE inhibitors $(\%)$ & 92 & 71 & NS & 67 & NS & 69 & NS \\
\hline Digitalis (\%) & 83 & 93 & NS & 53 & NS & 72 & NS \\
\hline Frusemide, dose (mg/day) & $100(88)$ & $74(43)$ & NS & $90(63)$ & NS & $82(54)$ & NS \\
\hline
\end{tabular}

Values are mean (SD) unless stated otherwise.

p Value expresses comparison $v$ the placebo group.

ACE, angiotensin converting enzyme; NYHA, New York Heart Association functional class. 


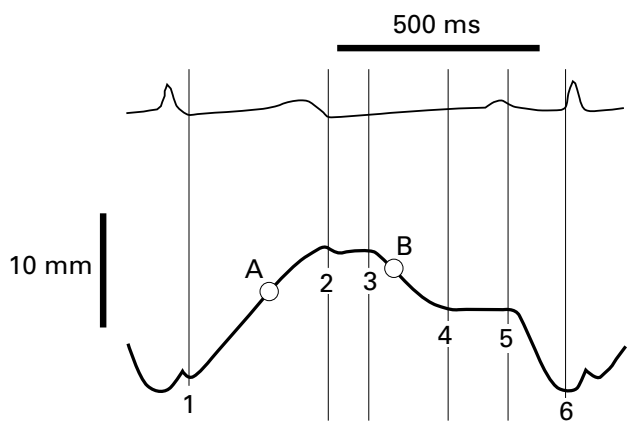

Figure 1 Schematic atrioventricular plane (AVP) curve and delineation of measured periods: (1) start of left ventricular contraction and ejection; (2) end of left ventricular contraction; (3) start of early rapid diastolic filling; (4) end of rapid filling, beginning of the diastasis; (5) end of diastasis, beginning of atrial contraction; (6) end of atrial contraction. (A) Maximum derivative of systolic ejection. (B) Maximum derivative of early rapid filling.

plane amplitude was calculated as the difference between point 1 and 2 . The diastasis period was measured as the time from point 4 to point 5 . In cases of tachycardia, the diastasis is abolished and there is a continuous downslope from point 3 to point 6 . In these cases the software extrapolated a midpoint between point 3 and 6, which was substituted for point 4. The systolic fractional shortening (FS) of the AV plane was calculated as the total systolic amplitude divided by the length of the long axis from the epicardial contour of ventricular apex to point 4 in the AV plane curve, expressing the total systolic amplitude normalised for the end diastolic length of left ventricular long axis (AVP-FS). Performance in the AV plane was calculated as the average of at least three beats. Where not stated otherwise, all results are reported as the average performance of the septal and the left lateral AV plane recordings. The average of diastolic and systolic distances from the transducer to the apical contour were used in the formula for AVP-FS calculation.

Normal values (mean (SD)) in our laboratory from 83 healthy individuals were as follows: left ventricular long axis, $87(9.4) \mathrm{mm}$; AVP-FS, 15.6 (3.5)\%; atrial filling fraction, 40 (15)\%; ejection time, 306 (25) ms; rapid filling time, 152 (44) ms; diastasis, 303 (125) ms; atrial filling time, 109 (17) ms; maximum ejection $\mathrm{dD} / \mathrm{dt}$ velocity, $6.4(1.6) \mathrm{cm} / \mathrm{s}$; maximum rapid filling $\mathrm{dD} / \mathrm{dt}$ velocity, $8.8(3.9) \mathrm{cm} / \mathrm{s}$. Normal short axis data were: interventricular septal thickening, $3.5(1.8) \mathrm{mm}$; posterior wall thickening, $6.9(1.7) \mathrm{mm}$; maximum interventricular septum ejection $\mathrm{dD} / \mathrm{dt}$ velocity, 3.7 (1.3) $\mathrm{cm} / \mathrm{s}$; maximum interventricular septum rapid filling $\mathrm{dD} / \mathrm{dt}$ velocity, $4.4(1.7) \mathrm{cm} / \mathrm{s}$; maximum posterior wall ejection $\mathrm{dD} / \mathrm{dt}$ velocity, $4.1(0.74) \mathrm{cm} / \mathrm{s}$; maximum posterior wall rapid filling $\mathrm{dD} / \mathrm{dt}$ velocity, 10.0 (3.9) $\mathrm{cm} / \mathrm{s}$. Mitral flow data were analysed from strip charts at $100 \mathrm{~mm} / \mathrm{s}$, as previously described. ${ }^{3}$

The study was approved by the ethics committee of the medical faculty, Göteborg University. Participating patients gave informed consent before inclusion in the study.
STATISTICAL METHODS

As the data were not normally distributed, non-parametric tests were used for statistical analysis. To avoid multiple statistical testing the patients on double blind metoprolol and those on open metoprolol treatment were evaluated together and compared with the placebo group. A within group repeated measurement analysis of variance (ANOVA) on ranks was used to evaluate changes over time. If significant, this test was followed by pairwise comparisons of differences between baseline and follow up investigations (Dunnett's method). A probability value of $\mathrm{p}<0.05$ was considered significant.

Multivariate forward stepwise regression analysis was used to evaluate load dependency of changes in non-invasive variables. The patients who were included in the invasive protocol and treated with metoprolol were analysed. A similar test was done to test which echocardiographic variable best correlated with fractional change in radionuclide ejection fraction over 12 months of metoprolol treatment. To test the ability of non-invasive variables to predict future changes in pulmonary capillary wedge pressure and global radionuclide ejection fraction, a multivariate forward stepwise regression analysis was used. The long term fractional changes in pulmonary capillary wedge pressure and ejection fraction, respectively, were entered as the dependent variable and the fractional changes in noninvasive short term (three month) data were used as independent variables.

Data are presented as mean (SD), and in the figures as mean with SEM. The statistics were performed on a Sigma Stat statistical software, version 2.0 (Jandel Corporation, San Rafael, California, USA).

\section{Results}

During the study there were three deaths: one placebo patient died after 45 days of treatment and was investigated at baseline only. This patient is not included in the present analysis. One metoprolol patient died suddenly after six months of treatment, and one placebo patient was withdrawn because of non-compliance after six months of treatment and died subsequently before the end of the study. Left ventricular function tended to be worse in the double blind study as compared with the patients on open metoprolol, although a lower age in the double blind metoprolol group was the only significant difference in comparison with the open treated group (table 1).

\section{SYSTOLIC AND REGIONAL LEFT VENTRICULAR} FUNCTION

The mean and regional AVP-FS improved significantly in the metoprolol group during the study, with no significant change in the placebo group (tables 2 and 3; fig 2). Most of the improvement had occurred after three months of treatment. The short axis recordings displayed poor septal mobility with no significant changes in interventricular septal wall thickening in either group (fig 2). As known from previous trials, the response to $\beta$ blocker treatment 
Table 2 Mean values of septal and left lateral atrioventricular plane motion

\begin{tabular}{|c|c|c|c|c|c|c|c|c|c|}
\hline & & Baseline & 3 months & $p$ Value & 6 months & $p$ Value & 12 months & $p$ Value & ANOVA \\
\hline Placebo/metoprolol (n) & & $12 / 29$ & $10 / 21$ & & $10 / 28$ & & $11 / 27$ & & \\
\hline \multirow[t]{2}{*}{ Heart rate $(\mathrm{ms})$} & $\mathrm{P}$ & $88(14)$ & $83(15)$ & - & $83(16)$ & - & $83(14)$ & - & 0.39 \\
\hline & M & $82(18)$ & $69(15)$ & $<0.05$ & $67(14)$ & $<0.05$ & $69(18)$ & $<0.05$ & $<0.001$ \\
\hline \multirow[t]{2}{*}{ Ejection time (ms) } & $\mathrm{P}$ & $203(48)$ & $224(29)$ & - & $215(48)$ & - & $220(44)$ & - & 0.95 \\
\hline & M & $223(37)$ & $240(33)$ & NS & $252(38)$ & $<0.05$ & $254(41)$ & $<0.05$ & 0.017 \\
\hline \multirow[t]{2}{*}{ Rapid filling time (ms) } & $\mathrm{P}$ & $58(13)$ & $69(29)$ & - & $68(269)$ & - & $66(22)$ & - & 0.62 \\
\hline & M & $73(38)$ & $103(57)$ & $<0.05$ & $113(52)$ & $<0.05$ & $117(67)$ & $<0.05$ & 0.004 \\
\hline \multirow[t]{2}{*}{ Total diastolic time (ms) } & $\mathrm{P}$ & $360(104)$ & 383 (137) & - & $398(150)$ & - & $390(116)$ & - & 0.13 \\
\hline & $M$ & $415(155)$ & $526(189)$ & $<0.05$ & $557(162)$ & $<0.05$ & $543(200)$ & $<0.05$ & 0.003 \\
\hline \multirow[t]{2}{*}{ AVP-FS (\%) } & $\mathrm{P}$ & $4.6(2.0)$ & $5.7(1.7)$ & - & $5.0(2.1)$ & - & $5.3(2.3)$ & - & 0.92 \\
\hline & M & $5.3(2.0)$ & $7.1(3.2)$ & $<0.05$ & $7.7(3.0)$ & $<0.05$ & $7.8(3.1)$ & $<0.05$ & 0.003 \\
\hline \multirow[t]{2}{*}{ Rapid filling amplitude (mm) } & $\mathrm{P}$ & $1.7(0.74)$ & $2.3(1.0)$ & - & $1.8(0.77)$ & - & $2.1(0.94)$ & - & 0.78 \\
\hline & M & $2.4(1.3)$ & $3.3(1.9)$ & $<0.05$ & $3.2(1.5)$ & $<0.05$ & $4.1(2.8)$ & $<0.05$ & $<0.001$ \\
\hline \multirow[t]{2}{*}{ Atrial filling fraction (\%) } & $\mathrm{P}$ & $68(27)$ & $74(27)$ & - & $70(43)$ & - & $58(30)$ & - & 0.24 \\
\hline & M & $65(20)$ & $59(22)$ & $<0.05$ & $61(22)$ & $<0.05$ & $58(22)$ & $<0.05$ & 0.031 \\
\hline \multirow[t]{2}{*}{ Maximum ejection, $\mathrm{dD} / \mathrm{dt}(\mathrm{cm} / \mathrm{s})$} & $\mathrm{P}$ & $2.9(0.9)$ & $2.9(0.8)$ & - & $2.9(0.8)$ & - & $2.8(1.0)$ & - & 0.62 \\
\hline & M & $3.1(0.8)$ & $3.6(1.4)$ & $<0.05$ & $3.9(1.3)$ & $<0.05$ & $3.9(1.2)$ & $<0.05$ & $<0.001$ \\
\hline \multirow[t]{2}{*}{ Maximum rapid filling, $\mathrm{dD} / \mathrm{dt}(\mathrm{cm} / \mathrm{s})$} & $\mathrm{P}$ & $3.7(1.3)$ & $3.8(1.3)$ & - & $3.4(0.9)$ & - & $3.7(1.6)$ & - & 0.67 \\
\hline & M & $4.4(1.8)$ & $4.8(2.8)$ & $<0.05$ & $4.2(2.0)$ & $<0.05$ & $5.2(2.8)$ & $<0.05$ & 0.012 \\
\hline
\end{tabular}

Values are mean (SD) unless stated otherwise. ANOVA column denotes significance of testing a repeated measurements analysis of variance test within respective group, followed by comparison with baseline value using Dunnett's test; -, no test performed.

AVP-FS, systolic fractional shortening in the atrioventricular plane; $\mathrm{M}$, metoprolol; $\mathrm{P}$, placebo.

Table 3 Echocardiographic short axis data

\begin{tabular}{|c|c|c|c|c|c|c|c|c|c|}
\hline & & Baseline & 3 months & $p$ Value & 6 months & $p$ Value & 12 months & $p$ Value & ANOVA \\
\hline Placebo/metoprolol (n) & & $12 / 29$ & $10 / 21$ & & $10 / 28$ & & $11 / 27$ & & \\
\hline \multirow{2}{*}{$\begin{array}{l}\text { Interventricular septum wall } \\
\text { thickening }(\mathrm{mm})\end{array}$} & $\mathrm{P}$ & $-0.1(1.2)$ & $0.3(0.77)$ & - & $0.3(0.97)$ & - & $0.3(1.3)$ & - & 0.87 \\
\hline & M & $0.5(1.2)$ & $0.7(1.5)$ & - & $0.7(1.8)$ & - & $0.6(1.1)$ & - & 0.35 \\
\hline \multirow[t]{2}{*}{ Posterior wall thickening $(\mathrm{mm})$} & $\mathrm{P}$ & $4.1(2.2)$ & $3.4(2.1)$ & - & $4.1(2.0)$ & - & $3.9(1.5)$ & - & 0.83 \\
\hline & M & $3.3(2.1)$ & $4.1(2.1)$ & $<0.05$ & $4.0(2.4)$ & $<0.05$ & $4.7(2.0)$ & $<0.05$ & 0.006 \\
\hline \multirow[t]{2}{*}{ Fractional shortening $(\%)$} & $\mathrm{P}$ & $13(2.1)$ & $13(2.9)$ & - & $14(4.7)$ & - & $14(4.9)$ & - & 0.71 \\
\hline & $M$ & $12(4.6)$ & $16(5.7)$ & $<0.05$ & $16(6.4)$ & $<0.05$ & $17(5.8)$ & $<0.05$ & $<0.001$ \\
\hline \multirow{2}{*}{$\begin{array}{l}\text { Maximum left ventricular ejection, } \\
\mathrm{dD} / \mathrm{dt}(\mathrm{cm} / \mathrm{s})\end{array}$} & $\mathrm{P}$ & $4.1(1.0)$ & $4.4(0.83)$ & - & $4.6(1.1)$ & - & $4.8(0.82)$ & - & 0.32 \\
\hline & M & $4.5(1.2)$ & $5.0(1.6)$ & - & $5.1(1.7)$ & - & $5.3(1.4)$ & - & 0.11 \\
\hline \multirow{2}{*}{$\begin{array}{l}\text { Maximum left ventricular filling, } \\
\mathrm{dD} / \mathrm{dt}(\mathrm{cm} / \mathrm{s})\end{array}$} & $\mathrm{P}$ & $8.3(2.6)$ & $8.8(2.2)$ & - & $8.3(2.4)$ & - & $8.9(2.4)$ & - & 0.95 \\
\hline & M & $8.3(2.6)$ & $9.1(4.0)$ & - & $8.2(2.8)$ & - & $8.9(3.0)$ & - & 0.74 \\
\hline \multirow{2}{*}{$\begin{array}{l}\text { Maximum interventricular septal wall } \\
\text { thickening, } \mathrm{dD} / \mathrm{dt}(\mathrm{cm} / \mathrm{s})\end{array}$} & $\mathrm{P}$ & $1.6(0.46)$ & $1.7(0.83)$ & - & $2.0(0.60)$ & - & $2.0(0.66)$ & - & 0.09 \\
\hline & $\mathrm{M}$ & $2.1(1.1)$ & $2.2(1.4)$ & - & $2.3(1.1)$ & - & $2.2(0.94)$ & - & 1.00 \\
\hline \multirow{2}{*}{$\begin{array}{l}\text { Maximum interventricular septal wall } \\
\text { thinning, } \mathrm{dD} / \mathrm{dt}(\mathrm{cm} / \mathrm{s})\end{array}$} & $\mathrm{P}$ & $1.3(0.91)$ & $1.6(0.90)$ & NS & $1.7(1.1)$ & NS & $2.2(1.0)$ & $<0.05$ & 0.02 \\
\hline & M & $1.7(1.2)$ & $2.0(1.8)$ & - & $2.0(1.3)$ & - & $2.3(1.4)$ & - & 1.00 \\
\hline \multirow{2}{*}{$\begin{array}{l}\text { Maximum posterior wall thickening, } \\
\mathrm{dD} / \mathrm{dt}(\mathrm{cm} / \mathrm{s})\end{array}$} & $\mathrm{P}$ & $3.1(0.87)$ & $3.2(1.1)$ & - & $3.2(0.89)$ & - & $3.2(0.91)$ & - & 0.95 \\
\hline & $M$ & $3.0(1.1)$ & $3.5(1.1)$ & - & $3.1(1.1)$ & - & $3.5(1.0)$ & - & 0.52 \\
\hline \multirow{2}{*}{$\begin{array}{l}\text { Maximum posterior wall thinning, } \\
\mathrm{dD} / \mathrm{dt}(\mathrm{cm} / \mathrm{s})\end{array}$} & $\mathrm{P}$ & $5.9(1.7)$ & $5.3(2.4)$ & - & $5.1(1.6)$ & - & $5.6(1.6)$ & - & 0.44 \\
\hline & M & $5.3(1.9)$ & $5.9(2.8)$ & - & $5.0(2.0)$ & - & $5.6(1.8)$ & - & 0.74 \\
\hline
\end{tabular}

ANOVA column denotes significance of testing a repeated measurements analysis of variance within respective group, followed by comparison with baseline value using Dunnett's test; -, no test performed.

was variable in the individual patient. The skewness in the data is illustrated in a box plot (fig 3), in which patients with different causes of heart failure are shown separately. The external longitudinal end diastolic dimension of the left ventricle was unchanged during treatment (placebo, 96 (10) to 93 (11) $\mathrm{mm}$,

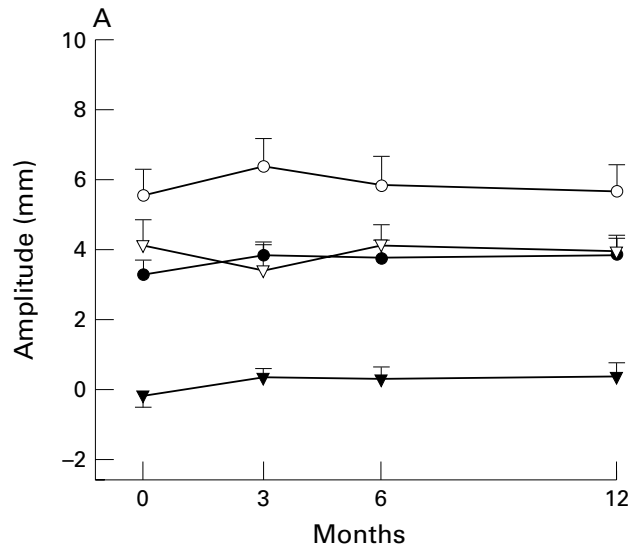

NS; metoprolol, 96 (11) to 97 (11) mm, NS) The sphericity index (end diastolic short axis to long axis ratio) was significantly reduced by 12 months in the metoprolol group, suggesting less sphericity (placebo, $0.93(0.07)$ to 0.96 (0.13), NS; metoprolol, $0.97(0.11)$ to 0.92 $(0.11), \mathrm{p}<0.05)$.

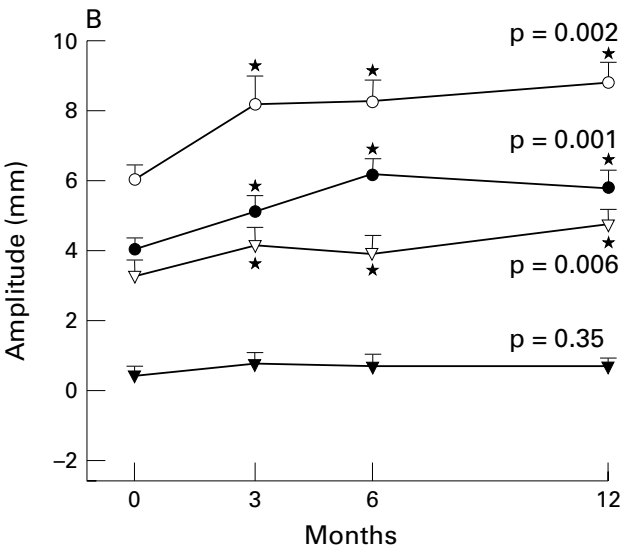

Figure 2 (A) Regional systolic amplitudes in patients on placebo. (B) Regional systolic amplitudes in patients on metoprolol treatment. Empty circles, left lateral atrioventricular plane (AVP); filled circles, septal AVP; empty triangles, posterior wall short axis; filled triangles, interventricular septum short axis. The p values of the analysis of variance test are shown to the right. ${ }^{*} p<0.05$ v baseline investigation. 


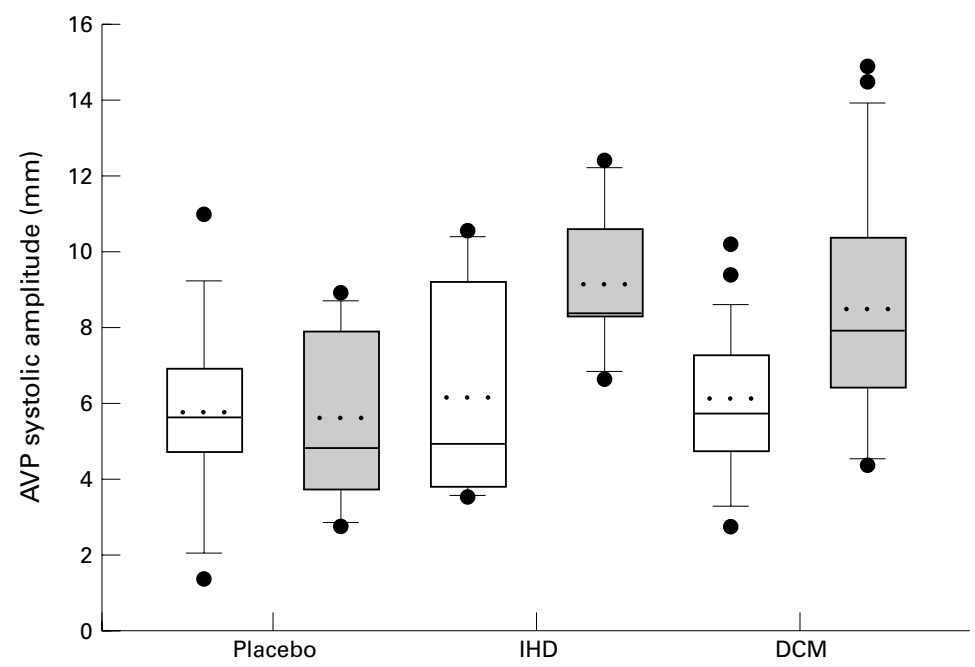

Figure 3 Box plots showing lateral atrioventricular plane (AVP) contraction amplitudes in patients on placebo (all dilated cardiomyopathy, $n=12$ ), in patients with ischaemic cardiomyopathy (IHD, all on open metoprolol, $n=6)$, and in patients with dilated cardiomyopathy (DCM) treated with metoprolol (open treatment, $n=9$; double blind treatment, $n=14$ ). Baseline (white boxes), six months follow up (grey boxes). The boxes encompass the 25th to the 75th centile, and the whiskers the 10th to the 90th centile. Dots denote values outside the 10th and 90th centiles. Solid lines in the boxes denote median values and dotted lines denote mean values.

DIASTOLIC FUNCTION AND RELATION TO FILLING PRESSURE

AV plane time intervals were significantly prolonged during $\beta$ blocker treatment. Concomitantly, there was an increase in the derivatives of the slope of contraction and rapid filling velocity (table 2). There were no significant changes in diastolic performance variables in short axis recordings (table 3 ).

Data on pulmonary capillary wedge pressure and radionuclide ejection fraction are shown in table 4 . The change in pulmonary capillary wedge pressure from baseline to six months was compared with changes in non-invasive data in a stepwise regression analysis. Whereas AV plane ejection time, maximum ejection velocity, and AVP-FS were univariately correlated, only the AVP-FS was independently and strongly correlated with the change in pulmonary capillary wedge pressure $(r=0.90$, $\mathrm{p}=0.001$; fig 4). Changes in stroke volume, systemic vascular resistance, or radionuclide ejection fraction did not correlate with the changes in wedge pressure. In a similar analysis, the change in radionuclide ejection fraction over 12 months was analysed by a stepwise regression, entering echocardiographic variables for the AV plane and the short axis, plus E wave mitral Doppler deceleration time. Only AVP-FS was significantly and independently associated with the change in ejection fraction $(r=0.76, \mathrm{p}<0.001$; fig 5).

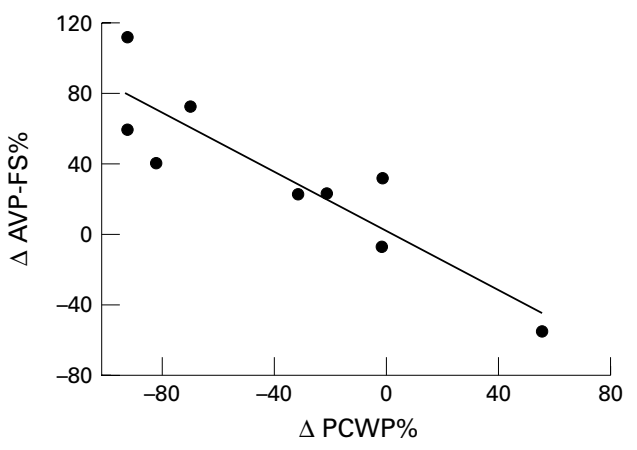

Figure 4 Linear correlation between systolic fractional shortening in the atrioventricular plane $(A V P-F S) v$. pulmonary capillary wedge pressure (PCWP) in patients treated with metoprolol in the invasive substudy over six months. In a multivariate stepwise regression analysis, the $A V P-F S$ was the only variable that was independently associated with the change in pulmonary capillary wedge pressure. $\triangle P C W \%=-4.35-0.96(\triangle A V P-F S \%), r=$ $0.90, p=0.001$.

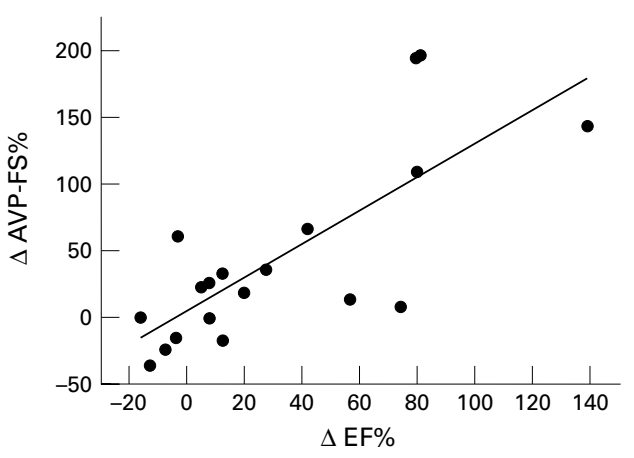

Figure 5 Linear correlation between systolic fractional shortening in the atrioventricular plane (AVP-FS) (over three months) versus change in radionuclide ejection fraction (EF) (over 12 months) in patients treated with metoprolol. In a multivariate stepwise regression analysis, the AVP-FS was the only echocardiographic variable that was independently associated with the future change in $E F$. $\triangle E F \%=12.3+0.46(\triangle A V P-F S \%), r=0.76, p<$ 0.001 .

To evaluate whether early changes in noninvasive variables could predict a future improvement in global cardiac performance, changes at three months were compared with the change in wedge pressure by six months, and radionuclide ejection fraction by 12 months. The stepwise multivariate procedure revealed that the change in pulmonary capillary wedge pressure could be predicted by a formula including three month changes in AVP-FS only: $\Delta$ pulmonary capillary wedge pressure $\%=-9.16-0.87(\Delta \mathrm{AVP}-\mathrm{FS} \%)$, $r=0.80, \mathrm{p}=0.017$. Further, the changes in radionuclide ejection fraction $(\mathrm{EF})$ by 12 months could be predicted by changes in AVP-FS and radionuclide ejection fraction by three months: $\triangle \mathrm{EF} \%=-6.3+0.58(\Delta \mathrm{AVP}-\mathrm{FS}$ $\%)+0.29(\Delta \mathrm{EF} \%)$. The individual coefficients

Table 4 Pulmonary capillary wedge pressure and radionuclide ejection fraction

\begin{tabular}{|c|c|c|c|c|c|c|c|c|}
\hline & & Baseline & 3 months & $p$ Value & 6 months & $p$ Value & 12 months & $p$ Value \\
\hline \multirow[t]{2}{*}{ Radionuclide ejection fraction (\%) } & $\mathrm{P}$ & $25(7.2)[12]$ & $29(9.2)[9]$ & 0.19 & 27 (11) [12] & 1.00 & $28(8.5)[11]$ & 0.90 \\
\hline & M & $27(11)[27]$ & $32(14)[20]$ & 0.004 & $34(15)[27]$ & 0.003 & $35(14)[22]$ & 0.005 \\
\hline \multirow[t]{2}{*}{ Pulmonary capillary wedge pressure $(\mathrm{mm} \mathrm{Hg})$} & $\mathrm{P}$ & $15(8.1)[12]$ & & & $9.2(6.0)[8]$ & 0.11 & $15(10)[9]$ & 0.25 \\
\hline & $M$ & $17(8.9)[14]$ & & & $8.8(7.5)[9]$ & 0.078 & $10(6.0)[9]$ & 0.13 \\
\hline
\end{tabular}

Numbers in square brackets denote the size of the group; $\mathrm{p}$ value expresses comparison $v$ baseline. $\mathrm{M}$, metoprolol; P, placebo. 


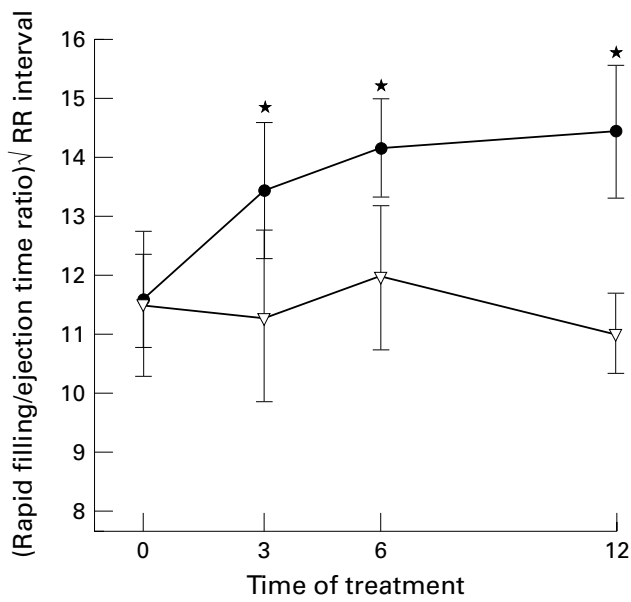

Figure 6 The atrioventricular plane (AVP) rapid filling time to ejection time ratio, corrected for heart rate by division with $Q_{1} Q_{2}$ interval. There was a significant prolongation of the rapid filling time to ejection time ratio in the metoprolol group, but no change in the placebo group. Filled circles, metoprolol; empty triangles, placebo. ${ }^{\star} p<0.05$ $v$ baseline.

were: $\triangle$ AVP-FS $\%, r=0.78, \mathrm{p}<0.001$; and $\Delta \mathrm{EF} \%, \quad r=0.34, \mathrm{p}=0.049$, respectively. Other non-invasive variables were not significantly related to future changes in pulmonary capillary wedge pressure and ejection fraction.

RELATION BETWEEN CHANGES IN HEART RATE AND NON-INVASIVE VARIABLES

Changes in heart rate were significantly related to changes in ejection time and rapid filling time $(r=0.72, \mathrm{p}<0.001)$ over 12 months of treatment. In contrast, neither ejection time maximum velocity $(r=0.16, \mathrm{p}=0.43)$ nor rapid filling time maximum velocity $(r=0.23$, $\mathrm{p}=0.25$ ) was significantly correlated with changes in heart rate. The AV plane rapid filling time divided by the square root of the $\mathrm{Q}_{1} \mathrm{Q}_{2}$ interval increased significantly in the metoprolol treated patients, from $2.56(1.05)$ at baseline to 3.69 (1.69) at 12 months $(p=0.001)$. The quotient of rapid filling time to ejection time, corrected for the $\mathrm{Q}_{1} \mathrm{Q}_{2}$ interval, also increased significantly in the metoprolol group and was unchanged in the placebo group (fig 6).

\section{Discussion}

This is the first study to show that recording the longitudinal motion pattern of the left ventricle can be used to evaluate drug effects on systolic and diastolic performance. Improvement in long axis contractions was more prominent than alterations in the short axis. Early changes in AV plane motion reflected future improvement in filling pressure and global ejection fraction.

AV PLANE AND DIASTOLIC FUNCTION

An intriguing finding was the increase in AV plane rapid filling velocity, because radionuclide studies have not been able to reveal amplitude differences during $\beta$ blockade treatment. ${ }^{12}{ }^{14}$ In contrast, it was not possible to detect any increase in short axis filling velocities. However, the absolute AV plane amplitudes during early diastole were small.
The high time resolution of $M$ mode echocardiography was probably a prerequisite for these observations. Radionuclide peak filling rate is difficult to assess in patients with small stroke volumes and high heart rates, because of the merging of early and late diastolic volume changes. In addition, the cardiac cycle is gated from the beginning of systole, which makes diastolic interpretation less reliable. It is possible that the absence of improved short axis filling rates attenuates the ability of the radionuclide method to detect improvement in long axis performance. The present results provide new data to enhance our understanding of ventricular recovery during $\beta$ blocker treatment. Although it has long been proposed that diastolic function would improve after $\beta$ blocker treatment, sparse data are available to prove this. ${ }^{3}$ The previous focus on circumferential motion might have caused an underestimation of the effects on subendocardial and longitudinal events. According to the suggestion that it is the longitudinal muscle fibres in particular that affect AV plane motion, recordings of the latter might be sensitive to changes in diastolic function.

In the present study we observed an increase in maximum early rapid filling velocity (by $18 \%$ from baseline to 12 months), despite a prolongation of the filling period. An increase in maximum velocity with longer filling implicates an increase in filling amplitude or enhanced acceleration of wall motion. Although the absolute changes in AV plane filling amplitudes were small-from 2.4 to $4.1 \mathrm{~mm}$ ( $71 \%$ increase) - the prolongation of the diastolic period was more prominent, from 73 to $117 \mathrm{~ms}$ (60\% increase). In addition, the atrial filling fraction decreased slightly (by $11 \%$ ). Thus we suggest that the prolongation of the early rapid filling period is the most important diastolic event during $\beta$ blocker treatment. These data are also in agreement with our previous findings of a lengthening of the $\mathrm{E}$ wave deceleration time. ${ }^{3}$ Prolongation of early rapid filling time might allow a longer time for calcium to be restored in the sarcoplasmic reticulum and thus ensure a more thorough and complete relaxation. An increase in sarcoplasmic reticular calcium stores would subsequently be the basis for the improvement in systolic performance. ${ }^{15}$ In an animal model it has been shown that $\beta$ blocker treatment can increase sarcoplasmic reticular $\mathrm{Ca}^{2+}$-ATPase activity. ${ }^{16}$

\section{INFLUENCE OF LOAD AND HEART RATE}

Whether or not $\beta$ blockers have a direct effect on the diastolic filling process could not be confirmed by the present data. There were simultaneous changes in both heart rate and filling pressure that also influence diastolic function. However, the increased length of the diastolic period is a direct effect of $\beta$ blockade, and it was proportionally more increased than the prolongation of the ejection period. We have previously shown that $\beta$ blocker treatment in congestive heart failure induces changes in cardiac function that are not merely dependent on a reduction in heart rate. ${ }^{31217}$ Treatment 
with $\beta$ blockers without vasodilating properties is not associated with afterload reduction. ${ }^{18} \mathrm{On}$ the contrary, a tendency towards an increase in blood pressure during metoprolol treatment might cause an increase in afterload. ${ }^{12} 18$ Although both systolic and diastolic improvements participate in the reduction in filling pressures, longitudinal contraction was most closely associated with changes in pulmonary capillary wedge pressure during $\beta$ blocker treatment. Apart from the contribution of longitudinal muscle fibres, it is possible that improved atrial function might contribute to the increase in AV plane amplitude. A possible explanation for the more prominent changes in the longitudinal contraction as compared with short axis contraction might be that $\beta$ blocker treatment is more effective in the subendocardial muscle fibres. It has been shown in an experimental study that longitudinal and circumferential muscle fibres are affected differently by $\beta$ blockade following myocardial infarction. ${ }^{19}$ Further, in congestive heart failure both $\beta$ receptor downregulation and noradrenaline (norepinephrine) depletion is enhanced in the subendocardial layers. ${ }^{20}{ }^{21}$ The $\beta$ receptors are upregulated, and postreceptor function is improved during metoprolol treatment..$^{23}$ These effects might explain the improvement in inotropic reserve that is found after long term metoprolol treatment. ${ }^{24}$

\section{LIMITATIONS}

In this investigation we only studied two left ventricular short axis regions and two AV plane regions. It is possible to study other positions in the AV plane.

The descent of the AV plane is perpendicular to the left ventricular long axis. It is usually easy to direct the $M$ mode beam at a right angle to the AV plane at the septal position, whereas at the lateral $\mathrm{AV}$ plane position the $\mathrm{M}$ mode beam often cuts the movements at a more acute angle. This might cause an angle error and less reliable estimations of the left lateral region.

In this study, we included a group of patients on open treatment to increase the numbers of patients investigated. While some differences were noted between the two groups of metoprolol treated patients, the trends were similar in both groups, suggesting similar effects of $\beta$ blockade in patients with varying aetiologies of heart failure and with varying degrees of ventricular dysfunction (fig 3 ).

\section{CONCLUSIONS}

AV plane contraction improvement after three months of metoprolol treatment was the best predictor of global improvement in radionuclide ejection fraction by 12 months. Additionally, AVP-FS was the only independent noninvasive variable associated with changes in filling pressure, suggesting that the basal portion of the left ventricle and the connected subendocardial muscle fibres are important for the recovery of myocardial function during $\beta$ blocker treatment.
The study was supported by the Swedish Heart Lung Foundation, the Swedish Medical Research Council, the Götebor Medical Society, and Astra Hässle AB, Mölndal, Sweden.

1 CIBIS-II Investigators and Committees. The cardiac insufficiency bisoprolol study II (CIBIS-II): a randomised trial. Lancet 1999;353:9-13.

2 The MERIT-HF Study Group. Effect of metoprolol CR/XL in chronic heart failure: Metoprolol CR/XL randomised intervention trial in congestive heart failure (MERIT-HF). Lancet 1999;353:2001-7.

3 Andersson B, Caidahl K, Di Lenarda A, et al. Changes in early and late diastolic filling patterns induced by long-term adrenergic beta-blockade in patients with idiopathic dilated cardiomyopathy. Circulation 1996;94: 673-82.

4 Henein MY, Priestley K, Davarashvili T, et al. Early changes in left ventricular subendocardial function after successful in left ventricular subendocardial function after

5 Alam M, Höglund C, Thorstrand C, et al. Effects of exercise on the displacement of the atrioventricular plane in on the displacement of the atrioventricular plane in
patients with coronary artery disease. A new echocardiopatients with coronary artery disease. A new echocardio-
graphic method of detecting reversible myocardial graphic method of detecting rever
ischemia. Eur Heart f 1991;12:760-5.

6 Willenheimer R, Cline C, Erhardt L, Israelsson B. Left ventricular atrioventricular plane displacement: an echocardiographic technique for rapid assessment of prognosis in heart failure. Heart 1997;78:230-6.

7 Tsakiris AG, Gordon DA, Padiyar R, et al. The role of displacement of the mitral annulus in left atrial filling and emptying in the intact dog. Can F Physiol Pharmacol 1978; 56:447-57.

8 Rogers WJ, Shapiro EP, Weiss JL, et al. Quantification of and correction for left ventricular systolic long-axis shortening by magnetic resonance tissue tagging and slice isolation. Circulation 1991;84:721-31.

9 Garcia MJ, Rodriquez L, Ares M, et al. Differentiation of constrictive pericarditis from restricitve cardiomyopathy: assessment of left ventricular diastolic velocities in longitudinal axis by Doppler tissue imaging. $7 \mathrm{Am}$ Coll Cardiol 1996;27:108-14.

10 Waagstein F, Bristow MR, Swedberg K, et al. Beneficial effects of metoprolol in idiopathic dilated cardiomyopathy. Lancet 1993;342:1441-6.

1 Andersson B, Blomstrom-Lundqvist C, Hedner T, et al. Exercise hemodynamics and myocardial metabolism during long-term beta-adrenergic blockade in severe heart failure. $\mathcal{F}$ Am Coll Cardiol 1991;18:1059-66.

12 Andersson B, Strömblad S-O, Lomsky M, et al. Heart rate dependency of cardiac performance in heart failure patients treated by metoprolol. Eur Heart 7 1999;20:57583.

13 Sahn DJ, DeMaria A, Kisslo J, et al. Recommendations regarding quantitation in M-mode echocardiography: results of a survey of echocardiographic measurements. results of a survey of echocarc
Circulation 1978;58:1072-83.

14 Quaife RA, Gilbert EM, Christian PE, et al. Effects of carvedilol on systolic and diastolic left ventricular performance in idiopathic dilated cardiomyopathy or ischemic cardiomyopathy. Am f Cardiol 1996;78:779-84

15 Morgan JP, Erny RE, Allen PD, et al. Abnormal intracellular calcium handling, a major cause of systolic and diastolic dysfunction in ventricular myocardium from patients with heart failure. Circulation 1990;81(suppl III):III-21-3.

16 Gwathmey JK, Kim CS, Hajiar RJ, et al. Cellular and molecular remodeling in a heart failure model treated with 90 .

17 Andersson B, Lomsky M, Waagstein F. The link between acute haemodynamic adrenergic beta-blockade and longterm effects in patients with heart failure. Eur Heart $\mathcal{F}$ 1993;14:1375-85.

18 Andersson B, Hamm C, Persson S, et al. Improved exercise hemodynamic status in dilated cardiomyopathy after beta-
adrenergic blockade treatment. 7 Am Coll Cardiol 1994;23: adrenergic

19 Birkeland S, Westby J, Grong K, et al. Effect of afterload and beta-adrenergic blockade on nonischemic myocardial contraction pattern. Am $\mathcal{F}$ Physiol 1992;263:H1716-23.

20 Beau SL, Saffitz JE. Transmural heterogeneity of norepinephrine uptake in failing human hearts. $\mathcal{F}$ Am Coll Cardiol 1994;23:579-85.

21 Beau SL, Tolley TK, Saffitz JE. Heterogeneous transmural distribution of beta-adrenergic receptor subtypes in failing human hearts. Circulation 1993;88:2501-9.

22 Waagstein F, Caidahl K, Wallentin I, et al. Long-term betablockade in dilated cardiomyopathy. Effects of short- and long-term metoprolol treatment followed by withdrawal and readministration of metoprolol. Circulation 1989;80: 551-63.

23 Böhm M, Deutsch HJ, Hartmann D, et al. Improvement of postreceptor events by metoprolol treatment in patients postreceptor events by metoprolol treatment in patients
with chronic heart failure. $\mathcal{F}$ Am Coll Cardiol 1997;30:9926 .

24 Heilbrunn SM, Shah P, Bristow MR, et al. Increased beta-receptor density and improved hemodynamic response to catecholamine stimulation during long-term metoprolol therapy in heart failure from dilated cardiomyopathy. Circulation 1989;79:483-90. 